\title{
Natural ferroelectric order near ambient temperature in the orthoferrite $\mathrm{HoFeO}_{3}$
}

\author{
K. Dey, ${ }^{1,2}$ A. Indra, ${ }^{1,3}$ S. Mukherjee, ${ }^{1}$ S. Majumdar, ${ }^{1}$ J. Strempfer, ${ }^{4}$ O. Fabelo,${ }^{5}$ E. Mossou, ${ }^{5}$ T. Chatterji, ${ }^{5}$ and S. Giri \\ ${ }^{1}$ School of Physical Sciences, Indian Association for the Cultivation of Science, Jadavpur, Kolkata 700032, India \\ ${ }^{2}$ Department of Physics, SBSS Mahavidyalaya, Goaltore, Paschim Medinipur, West Bengal 721128, India \\ ${ }^{3}$ Department of Physics, Srikrishna College, Bagula, Nadia, West Bengal 741502, India \\ ${ }^{4}$ Deutsches Elektronen-Synchrotron DESY, Notkestrasse 85, D-22607 Hamburg, Germany \\ ${ }^{5}$ Institut Laue-Langevin, 71 Avenue des Martyrs, CS 20156, 38042 Grenoble Cedex 9, France
}

(Received 23 April 2019; revised manuscript received 6 August 2019; published 26 December 2019)

\begin{abstract}
The current scenario in multiferroics demands a breakthrough discovery of promising materials after $\mathrm{BiFeO}_{3}$. Recently, the controversial discovery of room temperature ferroelectricity (FE) in $\mathrm{SmFeO}_{3}$ [Lee et al., Phys. Rev. Lett. 107, 117201 (2011); Kuo et al., Phys. Rev. Lett. 113, 217203 (2014)] inspires the investigation of $\mathrm{HoFeO}_{3}$. Here we report a natural ferroelectric order around $210 \mathrm{~K}\left(T_{\mathrm{FE}}\right)$ along the $c$ axis with a reasonably large polarization of $\sim 0.29 \mu \mathrm{C} / \mathrm{cm}^{2}$ for a $10 \mathrm{kV} / \mathrm{cm}$ poling field. The strong magnetoelectric coupling of $\sim 22 \%$ is observed at $210 \mathrm{~K}$ at $30 \mathrm{kOe}$, below which it decreases with a further increase in magnetic field. The synchrotron and neutron diffraction results confirm that a shift of $\mathrm{O}$ atoms along the $c$ axis, on the polar $P b^{\prime} n^{\prime} 2_{1}$ magnetic space, causes the $\mathrm{FE}$ in $\mathrm{HoFeO}_{3}$. The potential results of reasonably high ferroelectric polarization associated with a strong magnetoelectric coupling at higher temperature are fascinating and create a renewed attention for searching promising candidates with a natural ferroelectric order and higher $T_{\mathrm{FE}}$ in the rest of the $R \mathrm{FeO} \mathrm{O}_{3}$ series.
\end{abstract}

DOI: $10.1103 /$ PhysRevB.100.214432

\section{INTRODUCTION}

Spin dependent electronics is identified to be the nextgeneration technology that prevails over the current generation of electronics solely based on the charge degrees of freedom. In particular, the control of magnetism by applying the external electric field is a great challenge in materials science for developing low power spintronic devices [1-4]. Multiferroics, belonging to a chemically single phase rare class of materials, are promising to meet the above criterion [5]. The magnetoelectric (ME) coupling, as a result of cross coupling between magnetic and ferroelectric (FE) orders, leads to the tunability of magnetic polarization by the applied electric fields $(E)$. In order to explore it technologically, this functionality should be observed close to room temperature. Therefore, searching for the new promising materials after $\mathrm{BiFeO}_{3}$ is one of the prime focuses in multiferroics, where the large FE polarization $(P)$ associated with a reasonable ME coupling is desirable close to room temperature [6]. The cupric oxide, $\mathrm{CuO}$ (tenorite), is an another promising inorganic material that exhibits a spontaneous polar order, however, in a limited temperature region of $213-230 \mathrm{~K}[7,8]$.

Recently, few members of the rare earth $(R)$ ferrite $\left(R \mathrm{FeO}_{3}\right)$ has been found to hold the great promise of room temperature multiferroic order in the artificial hexagonal structures [9-12]. The ferroelectricity at room temperature is observed for epitaxially grown $\mathrm{YbFeO}_{3}$ [9], $\mathrm{GaFeO}_{3}$ [10], and $\mathrm{LuFeO}_{3}$ [11] thin films, where a hexagonal heterostructure has been adopted in all the cases. More recently, the interface straininduced ferroelectricity has also been proposed for $\mathrm{SmFeO}_{3}$ films, which was epitaxially grown on a $[001] \mathrm{SrTiO}_{3}: \mathrm{Nb}$ substrate with a proposed rhombohedral or hexagonal structure [12]. The compound $\mathrm{SmFeO}_{3}$ has been the center of attention, when the spin-canting driven natural ferroelectricity was reported above room temperature along the crystallographic $b$ axis [13]. On the contrary, a $G$-type collinear antiferromagnetic (AFM) structure has been established and suggested for the nonexistent ferroelectricity in $\mathrm{SmFeO}_{3}$ [14]. More recent results on polycrystalline $\mathrm{SmFeO}_{3}$ again reported an evidence of the spontaneous electric polarization at $173 \mathrm{~K}$, as confirmed by the polarization loop $(P-E)$ measurements [15]. In fact, the first-principles calculations proposed a possible occurrence of electric polarization along the $b$ axis associated with the $P m c 2_{1}$ ground state, where the exchange striction mechanism involved a polar displacement of the oxygen ions correlated with the proposed ferroelectricity [16]. These contradictory results create arguments on the occurrence of intrinsic ferroelectricity in $\mathrm{SmFeO}_{3}$. Unlike the abovementioned $R \mathrm{FeO}_{3}$, the $\mathrm{AFM} \mathrm{GdFeO}_{3}$ showed a FE transition along the crystallographic $c$ axis at the Gd ordering temperature of $2.5 \mathrm{~K}[17,18]$, which was further supported by the $a b$ initio calculations considering an exchange striction between adjacent $\mathrm{Fe}$ and $\mathrm{Gd}$ atoms [19]. Below the Dy ordering transition at $3.5 \mathrm{~K}$, the ferroelectricity was also observed for $\mathrm{DyFeO}_{3}$ with a magnetic field $(H)$ applied along the $c$ axis [20]. Importantly, the application of magnetic field causes a change of $\mathrm{Fe}$ spin configuration to the original high temperature $G_{x} F_{z}$ structure from $A_{x} G_{y}$ and points to the fact that the ferroelectric order in $\mathrm{DyFeO}_{3}$ appears in the high temperature spin structure.

The compound of our interest, the orthoferrite $\mathrm{HoFeO}_{3}$, exhibits a long range magnetic order governed by the $\mathrm{Fe}-\mathrm{Fe}$, $R-\mathrm{Fe}$, and $R-R$ interactions. The $\mathrm{Fe}$ spins of the compound order antiferromagnetically $\left(T_{N}\right)$ around $647 \mathrm{~K}$, which is followed by two more transitions driven by the $R$-Fe and $R-R$ interactions with decreasing temperature [21-25]. As a result of the $R-\mathrm{Fe}$ interaction, the $\mathrm{Fe}$ spins reorient along the 
$c$ axis below $\sim 51 \mathrm{~K}$ and transform to a $G_{z} F_{x}$ phase from $G_{x} F_{z}$ in Bertaut's notation. The Ho order was further proposed to happen around $\sim 3.3 \mathrm{~K}$ from the heat capacity, magnetization results, and Mössbauer studies for $\mathrm{HoFeO}_{3}$ [26-28].

In this article we report the onset of polar order along the crystallographic $c$ axis below $\sim 240 \mathrm{~K}$ for $\mathrm{HoFeO}_{3}$ with a FE transition $\left(T_{\mathrm{FE}}\right)$ at $\sim 210 \mathrm{~K}$. Importantly, the $P$ appears in the magnetic $G_{x} F_{z}$ state. This is exactly the same magnetic $G_{x} F_{z}$ state of $\mathrm{DyFeO}_{3}$ with nonzero $H$ and $\mathrm{GdFeO}_{3}$ with $H=0$ [17], where ferroelectricity was observed at very low temperatures below the respective rare earth ordering temperatures. Here the value of $P$ is significantly higher as $\sim 0.29 \mu \mathrm{C} / \mathrm{cm}^{2}$ for a $10 \mathrm{kV} / \mathrm{cm}$ poling field. A considerable increase ( $\sim 23 \%$ at $T_{\mathrm{FE}}$ for $30 \mathrm{kOe}$ ) of $P$ is noted at higher temperature, pointing to a significant magnetoelectric coupling. Synchrotron diffraction studies indicate that the structural changes are associated with the FE ordering, as observed from the $[11 l]$ scan. The amplitude refinement of the neutron diffraction results confirm that the shift of the apex $\mathrm{O}$ atoms of the $\mathrm{FeO}_{6}$ octahedra along $c$ axis in the $\mathrm{Pbn} 2_{1}$ polar structure correlates the ferroelectricity. The change of slope is observed in the thermal variation of [011] magnetic reflection around $T_{\mathrm{FE}}$, which indicates the possible magnetoelectric coupling for $\mathrm{HoFeO}_{3}$.

\section{EXPERIMENTAL DETAILS}

The $\mathrm{HoFeO}_{3}$ single crystals were grown by Barilo and Zhigunov in Minsk, Belarus using flux method. A single crystal neutron diffraction study was performed on a neutronsize crystal, which was glued on a vanadium pin and placed on the self-dedicated low-temperature Displex device [29]. Two full data sets were collected at 200 and $250 \mathrm{~K}$ at the D19 diffractometer (ILL, Grenoble) operating in high resolution mode with a wavelength of $0.94822 \AA$. Data sets consist of omega scans at selected $\chi$ and $\phi$ positions to obtain a completeness above $90 \%$. The data collection was performed using the MAD program. The reflection data were indexed with the ILL program PFIND and integrated with the RETREAT software [30] from the ILL program suite. The correction for attenuation was carried out with the ILL program D19ABS [31]. Neutron diffraction was further performed on a crystal with a $2.36 \AA$ wavelength in the D10 diffractometer of ILL. The crystal was mounted on the cold tip of the D10 cryostat with its [100] axis approximately parallel to the $\omega$ axis of the diffractometer. A single crystal synchrotron diffraction study was performed at beamline P09/PETRA III at DESY, Germany [32]. The samples were mounted in a He-flow magnet cryostat covering the temperature range from 2 to $300 \mathrm{~K}$ with the cryostat mounted on a horizontal Psi diffractometer. The experiment was conducted at photon energies significantly below the L2/3 absorption edges of the rare earth elements to avoid fluorescence. Dielectric permittivity was recorded in a E4980A LCR meter (Agilent Technologies, USA) equipped with a PPMS-II system (Quantum Design, USA). The pyroelectric current was recorded using an electrometer (Keithley, model 6517B) at a constant temperature sweep rate. The $P-E$ loops were recorded using a FE loop tracer (Radiant Technology, USA). All electrical contacts were fabricated using an air drying silver paint. Heat capacity was measured in a PPMS-I
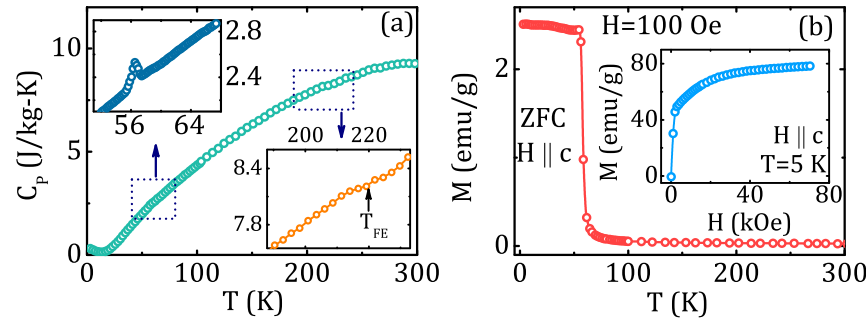

FIG. 1. Thermal variations of (a) heat capacity $\left(C_{P}\right)$ and (b) magnetization $(M)$ along the $c$ axis for $\mathrm{HoFeO}_{3}$. Upper and lower insets of (a) magnify the low- $T$ and high- $T$ regions, respectively. Inset of (b) shows a magnetization curve at $5 \mathrm{~K}$.

system (Quantum Design, USA). The DC magnetization was measured in a commercial magnetometer of Quantum Design (MPMS, evercool).

\section{EXPERIMENTAL RESULTS AND DISCUSSIONS}

Heat capacity $\left(C_{P}\right)$ in zero-field with temperature $(T)$ is depicted in Fig. 1(a) for $\mathrm{HoFeO}_{3}$. Upper and lower insets provide the low- $T$ and high- $T$ regions, highlighting the spin reorientation transition at $56 \mathrm{~K}[21,22,24,26-28]$ and an anomaly close to $T_{\mathrm{FE}}$, respectively. A change of slope in $C_{P}(T)$ is observed around $T_{\mathrm{FE}}$, which is correlated with the symmetry breaking, also confirmed by the synchrotron and neutron diffraction results. The sharp rise in ZFC magnetization $(M)$ at $56 \mathrm{~K}$ points to a spin reorientation transition, while any noticeable change is absent around $T_{\mathrm{FE}}$, as depicted in Fig. 1(b) for $H=100 \mathrm{Oe}$ along the $c$ axis. A magnetization curve at $5 \mathrm{~K}$ is depicted in the inset of Fig. 1(b). It shows an initial sharp rise with $H$, followed by a saturating trend above $\sim 40 \mathrm{kOe}$.

Dielectric permittivity $(\epsilon)$ is measured along the $c$ axis with $T$ at different frequencies $(f)$ in the range of $2-20 \mathrm{kHz}$. The real $\left(\epsilon^{\prime}\right)$ components of $\epsilon$ are plotted with $T$ in Fig. 2(a). Any convincing signature of $T_{\mathrm{FE}}$ is absent. The absence of
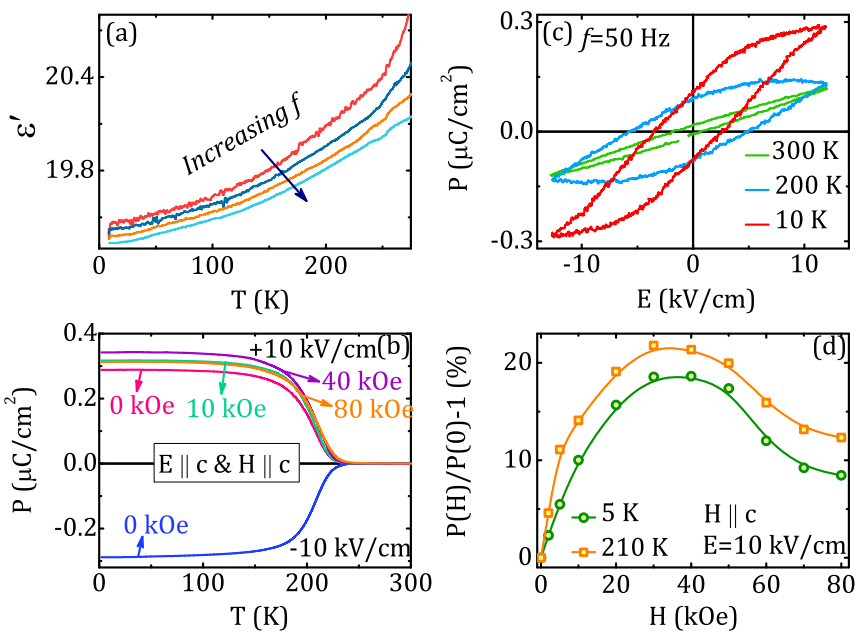

FIG. 2. Thermal variations of (a) real $\left(\epsilon^{\prime}\right)$ part of dielectric permittivity at different $f=2,5,10$, and $20 \mathrm{kHz}$, (b) electric polarizations $(P)$ for $E= \pm 10 \mathrm{kV} / \mathrm{cm}$ and with selected $H$, and (c) $P-E$ loops at 10, 200, and $300 \mathrm{~K}$. (d) Percentage of change of polarization with $H$ at 5 and $210 \mathrm{~K}$. 
signature in the high temperature may be attributed to the overlapping of the intrinsic component with the extrinsic components such as the grain boundary and the sample-electrode interface effects [33-35]. Appearance of a ferroelectric order is first detected along the $c$ axis from the pyroelectric current measurements, while the sample is cooled from different poling temperatures $\left(T_{\text {pole }}\right)$, as shown in Fig. S1 of the Supplemental Material (SM) [36] (see, also, Refs. [37-40] therein). For all $T_{\text {pole }}$, a peak around $\sim 210 \mathrm{~K}$ is always detected and confirms genuine occurrence of a polar order. Time integrated pyroelectric current measurements provide $P(T)$ for the \pm 10 $\mathrm{kV} / \mathrm{cm}$ poling field along the crystallographic $c$ axis, which is depicted in Fig. 2(b). Our attempts fail to detect the ferroelectricity along the $a$ and $b$ axes. The result is similar to the observed ferroelectricity along the $c$ axis for $\mathrm{GdFeO}_{3}$ [17] and $\mathrm{DyFeO}_{3}$ [20]. Reversal of $P$ for $E=-10 \mathrm{kV} / \mathrm{cm}$ corroborates the ferroelectricity. Figure 2(c) depicts the $P-E$ loops at 10,200 , and $300 \mathrm{~K}$. At $300 \mathrm{~K}$ the loop ascribes to the lossy current. The value of $P$ is $\sim 0.29 \mu \mathrm{C} / \mathrm{cm}^{2}$ at $10 \mathrm{~K}$, which is comparable to those reported for the promising orthoferrites [17] and manganites [41]. The value of $P$ decreases at $200 \mathrm{~K}$ in accordance with the $T$ variation of $P(T)$. As shown in Fig. 2(b), the $P(T)$ is strongly influenced by $H$ along the $c$ axis, pointing to a significant ME coupling. The percentage of changes in $P$ defined as $\Delta P(\%)=[P(H) / P(0)-1] \times 100$ at $5 \mathrm{~K}$ and $T_{\mathrm{FE}}$ are depicted as a function of $H$ in Fig. 2(d). Here $P(0)$ is recorded at zero field. $\Delta P(\%)$ increases with $H$ and a maximum is reached for both temperatures in the field range of 30-40 kOe, above which it decreases till $80 \mathrm{kOe}$. We note that the changes in $\Delta P(\%)$ at $5 \mathrm{~K}$ may be correlated with the magnetization curve at $5 \mathrm{~K}$, as shown in the inset of Fig. 1(b) [42]. Here the increase of $\Delta P(\%)$ is associated with the increase of $M$ initially and the decrease of $\Delta P(\%)$ is noted above $\sim 40 \mathrm{kOe}$, above which a saturating trend of $M$ is observed in the magnetization curve. The maximum value is achieved as high as $\sim 23 \%$ at $T_{\mathrm{FE}}$ for $H=30 \mathrm{kOe}$.

To investigate the structural correlation to the observed FE order, the synchrotron diffraction study [32] is performed over a temperature range of 5-300 K. The changes in the peaks at selected $T$ are shown in Fig. 3(a). The peak positions and integrated intensities are depicted with $T$ in Figs. 3(b) and 3(c), respectively. A maximum is observed in the peak position, which is slightly above $T_{\mathrm{FE}}$. This points to a significant change in the lattice parameters around $T_{\mathrm{FE}}$, because the change in $l$ is inversely proportional to the lattice constant $c$. The inset of Fig. 3(b) depicts the $T$ variation of $c(T)$, scaled by the value at $300 \mathrm{~K}[c(T) / c(300 \mathrm{~K})]$. An anomalous thermal expansion of $c(T) / c(300 \mathrm{~K})$ is observed around $T_{\mathrm{FE}}$. With decreasing $T$ from $300 \mathrm{~K}$, the integrated intensity remains unchanged till $T_{\mathrm{FE}}$, below which it decreases, exhibiting a "dip." Below $\sim 200 \mathrm{~K}$ it increases down to the low temperature, exhibiting another dip close to $\sim 55 \mathrm{~K}$. This is close to the spin reorientation transition, pointing to a magnetoelastic coupling. Here the change in the integrated intensities around $T_{\mathrm{FE}}$ is significant, because it involves a change in the scattering cross section and may point to a possible structural transition, as adequately reported for the isostructural transition [43-45] as well as symmetry lowering $[33,46-48]$ around the FE ordering.

We note that a $G$-type $\mathbf{k}=0$ AFM structure has been proposed $[21,24,25]$, around which ferroelectricity occurs in
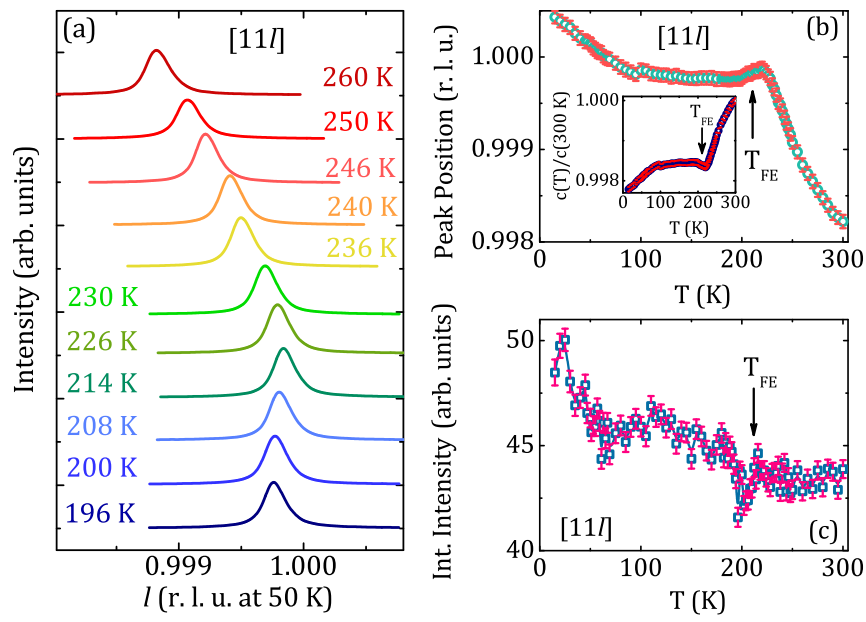

FIG. 3. (a) The $[11 l]$ scan at selected temperatures around $T_{\mathrm{FE}}$. The $T$ variations of (b) peak positions and (c) integrated intensity of $[11 l]$ scan. Inset of (b) shows the variation of $c(T) / c(300 \mathrm{~K})$ with $T$.

$\mathrm{HoFeO}_{3}$. Thus, the inverse Dzyaloshinskii-Moriya (DM) interaction does not contribute to the observed ferroelectricity. To resolve this, a neutron diffraction study is performed on a $\mathrm{HoFeO}_{3}$ crystal at two temperatures, above $(250 \mathrm{~K})$ and below (200 K) $T_{\mathrm{FE}}$. Refinements are performed by taking into account both the displacive and magnetic symmetry modes using a single crystal Amplitude Fullprof program, where the pcr file has been created using the ISODISTORT suit [49]. We further note that the $P b n 2_{1}$ has the highest symmetry among possible noncentrosymmetric structures, which is settled by the ISODISTORT software [50]. At $250 \mathrm{~K}$ the Pbnm space group is used as a parent space group. At $200 \mathrm{~K}$ the refinements are performed using both $P b n m$ and $P b n 2_{1}$ as the parent space groups, where the polar $P b n 2_{1}$ is a subgroup of Pbnm. We note that the R-factor improves from 3.96 to 3.76 for the refinement using $P b n 2_{1}$ as a parent space group at $200 \mathrm{~K}$, which has been considered in the reported neutron results.[24,25] Although the symmetry is lowered, here the final refinement is stable and converges to reasonable values. More importantly, it explains the ferroelectricity.

Out of four different Shubnikov space groups, such as $P b n 2_{1}, P b n^{\prime} 2_{1}^{\prime}, P b^{\prime} n 2_{1}^{\prime}$, and $P b^{\prime} n^{\prime} 2_{1}$, the last one only reproduces the magnetic structure at $200 \mathrm{~K}[24,25]$. Only two irreducible representations are needed to distort the $P b n 2_{1}$ parent space group into the $P b^{\prime} n^{\prime} 2_{1}$ Shubnikov space group. Here we discuss only GM1+, because it is the only displace mode in our system. In this Shubnikov group there are 15 possible modes vectors acting on the nuclear part at $200 \mathrm{~K}$, where only four modes such as A1, A4, A5, and A9 are active for the refinement using $P b n 2_{1}$, as a parent space group. The details are further discussed in the SM [36]. The amplitude A1, operating on the Ho(III) atoms with an amplitude of 0.0021(6) $\AA$, gives rise to a weak shift in the $a b$ plane. However, this amplitude cannot be responsible for the global ferroelectricity, because the polarization due to the displacement of a Ho atom is compensated by the same displacement with an opposite direction by the other Ho atom inside the unit cell, as more clearly depicted by the green small arrows in Fig. 4(a). A similar behavior is observed for the 

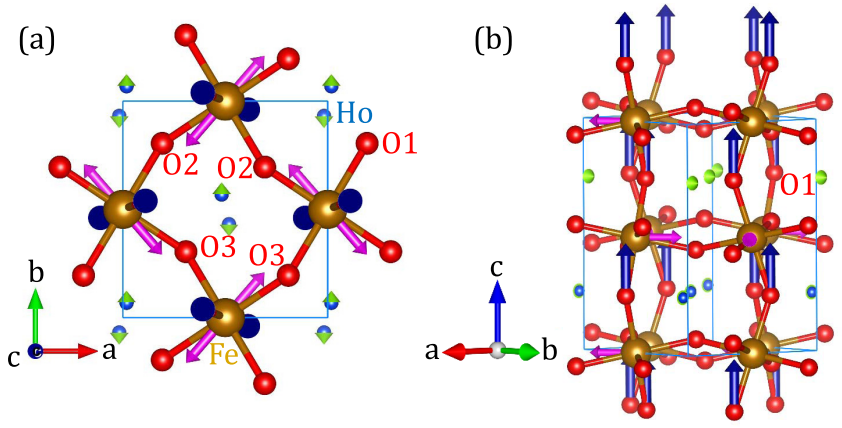

FIG. 4. The atomic displacements are depicted in different directions. (a) The directions of small Ho (green) and considerable $\mathrm{Fe}$ (pink) displacements for the projection along the $c$ axis. (b) The displacement of $\mathrm{O} 1$ atoms (deep blue) along the $c$ axis is shown. A fix scale of 10 is used for all the active modes.

Fe atoms. In this case two different amplitudes, A4 and A5 with amplitudes of $-0.011(3)$ and $-0.009(2) \AA$, are present. The combination of these two modes also produces a shift of the iron atoms in the $a b$ plane close to the Fe-O2 and $\mathrm{Fe}-\mathrm{O} 3$ bonds [51]. However, as in the case of Ho, these vectors are strictly compensated within the unit cell, as shown by the pink arrows in Fig. 4(a). Therefore, the Fe displacement does not contribute to the global polarization. Out of four amplitudes, the last amplitude, A9, of -0.022(5) $\AA$ acts on $\mathrm{O} 1$ of the $\mathrm{FeO}_{6}$ octahedra, producing a global shift along the crystallographic $c$ axis. This displacement has the same direction for all the $\mathrm{O} 1$ within the unit cell, as clearly depicted by the deep blue arrows in Fig. 4(b) and is accountable for the macroscopic electric polarization. This result is consistent with the anomalous expansion in $c(T) / c(300 \mathrm{~K})$ near $T_{\mathrm{FE}}$. Using the atomic displacement of $\mathrm{O} 1$, the value of $P$ is $\sim 1.2 \mu \mathrm{C} / \mathrm{cm}^{2}$ using a simplified formula $P=1 / V\left[\sum_{i}\left(m_{i} \Delta z_{i} Z_{i}\right) e\right]$, where $m_{i}$ is the crystallographic site multiplicity, $\Delta z_{i}$ is the displacement along the polar axis (here crystallographic $c$ axis), $Z_{i} e$ is the charge, and $V$ is the unit cell volume. The calculated value is $\sim 3$ times higher than the value of $P$, as obtained for the poling field of $10 \mathrm{kV} / \mathrm{cm}$. Instead of point charge model, the Berry phase method using a first-principles calculation would provide a more realistic value of $P$ [52]. We note that the proposed polar $P b n 2_{1}$ structure has also been proposed for a few members of the $R \mathrm{CrO}_{3}$ series $[33,48,53]$ and $\mathrm{SmFeO}_{3}$ [15] for accounting for ferroelectricity.

To understand the possible origin of the ferroelectric order, magnetic reflections are recorded as a function of temperature. The variations of the integrated intensities of the magnetic reflections along selected directions are shown in Fig. S5 of the SM [36]. Out of all the recorded magnetic reflections, the [011] reflection is the most sensitive for the proposed magnetic structure, as shown in Fig. S4 of the SM [36]. The temperature variation of the [011] reflection is depicted in Fig. 5(a), pointing to a change of slope around $T_{\mathrm{FE}}$. The sharp fall of intensity around $\sim 55 \mathrm{~K}$ further confirms the spin reorientation transition, as also evident in the magnetization and heat capacity results [24]. The proposed magnetic structure at $200 \mathrm{~K}$, described on $P b^{\prime} n^{\prime} 2_{1}$, is shown in Fig. 5(b), which is projected in the $a b$ plane. As obtained from the refinement, only one component of the three magnetic amplitudes is
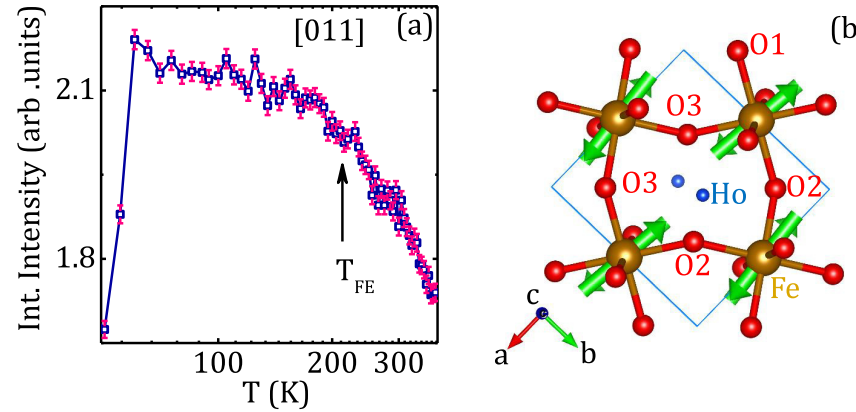

(b)

FIG. 5. $T$ variations of integrated intensity of the magnetic [011] reflections. (b) Proposed magnetic structure projected in the $a b$ plane.

dominant and the value is $3.72(3) \mu_{B}$ along the $a$ axis at $200 \mathrm{~K}$. The other two components at $200 \mathrm{~K}$ are $0.39(3)$ and $0.274(2)$ $\mu_{B}$ along the $b$ and $c$ axes, respectively. Each magnetic moment is not only antiferromagnetically coupled with the neighbors within the $a b$ plane but also along the $c$ axis, giving rise to a global antiferromagnetic behavior, as depicted in Fig. $\mathrm{S} 4$ of the SM [36]. We note that above $T_{\mathrm{FE}}$ a similar refinement at $250 \mathrm{~K}$ provides the magnetic amplitudes, as 3.79(3), $0.39(3)$, and $0.3(2) \mu_{B}$ along the $a, b$, and $c$ axes, respectively, where $P b^{\prime} n^{\prime} m$ is used for the refinement. The slight changes in magnetic amplitudes below and above $T_{\mathrm{FE}}$ are consistent with the slope change in the [011] reflection intensity around $T_{\mathrm{FE}}$. Using the values of the coordinates, as obtained from the refined nuclear structure, the $\mathrm{Fe}-\mathrm{Fe}$ distances are determined at 200 and $250 \mathrm{~K}$. The values of Fe-Fe distance along the $a$ axis $\left(d_{\mathrm{Fe}-\mathrm{Fe}}^{a}\right)$ are 5.2731 and $5.2767 \AA$ at 250 and $200 \mathrm{~K}$, respectively. Similarly, the values of $d_{\mathrm{Fe}-\mathrm{Fe}}^{b}$ are 5.5898 and $5.5957 \AA$, and for $d_{\mathrm{Fe}-\mathrm{Fe}}^{c}$ the values are 4.0002 and $3.9960 \AA$ at 250 and $200 \mathrm{~K}$, respectively. Thus, the exchange coupling is much stronger along the $c$ axis due to smaller values of $d_{\mathrm{Fe}-\mathrm{Fe}}^{c}$ compared to the values in the $a b$ plane. We further note that the change in the $d_{\mathrm{Fe}-\mathrm{Fe}}$ of $\sim 0.07 \%$ is observed in the $a b$ plane, which is smaller than the change of $\sim 0.11 \%$ along the $c$ axis for the change in temperature from 250 to $200 \mathrm{~K}$, pointing to the stronger structural distortions along the $c$ axis. Furthermore, the exchange interaction is ferromagnetic along the $a$ and $b$ axes and AFM along the $c$ axis, as shown in Fig. S4 of the SM [36]. As depicted in Fig. 4, a shift of $\mathrm{O} 1$ atom is observed along the $c$ axis and this leads to the inequivalent changes in the Fe-O1 bond lengths along the $c$ axis, giving rise to a significant change in the $\mathrm{Fe}-\mathrm{O} 1-\mathrm{Fe}$ superexchange path. A small change in the Fe-O1-Fe bond angle is also observed. The change in both the Fe-Fe bond length together with the change in the $\mathrm{Fe}-\mathrm{O} 1-\mathrm{Fe}$ superexchange path points to the evidence of significant exchange striction mechanism in the $c$ axis, along which the macroscopic electric polarization is observed. We note that similar exchange striction mechanism driven ferroelectricity has been addressed for the type-II multiferroics such as, $\alpha-\mathrm{Cu}_{2} \mathrm{~V}_{2} \mathrm{O}_{7}$ [54] and $\mathrm{GdMn}_{2} \mathrm{O}_{5}$ [55]. Thus, we propose a possible exchange striction mechanism to correlate the emergence of the ferroelectric order in $\mathrm{HoFeO}_{3}$.

In conclusion, ferroelectric order is observed near ambient temperature with $T_{\mathrm{FE}}$ at $210 \mathrm{~K}$ for $\mathrm{HoFeO}_{3}$. The reasonably high value of the electric polarization $\left(\sim 0.29 \mu \mathrm{C} / \mathrm{cm}^{2}\right)$ associated with a strong magnetoelectric coupling at low magnetic 
field attracts the community. Synchrotron and neutron diffraction studies of the single crystal propose that the breaking of space inversion symmetry in polar $P b n 2_{1}$ structure correlates with the ferroelectricity. More specifically, the apex oxygen displacement of the $\mathrm{FeO}_{6}$ octahedra engineers the ferroelectric polarization along the crystallographic $c$ axis. A possible exchange striction mechanism is suggested for interpreting the ferroelectricity in $\mathrm{HoFeO}_{3}$. This work contributes a significant progress in searching new type-II multiferroics with a hightemperature magnetoelectric coupling.

\section{ACKNOWLEDGMENTS}

The authors would like to thank J. Rodríguez-Carvajal for discussions and integrating the Amplitude refinement program to the Fullprof suite. S.G. acknowledges the financial support from DST, India (Project No. SB/S2/CMP029/2014), from BRNS, India for the neutron diffraction studies at ILL, France, and from DST-DESY project (No. I-20140424) for the synchrotron diffraction experiment at DESY, Germany.
[1] N. A. Spaldin, S.-W. Cheong, and R. Ramesh, Phys. Today 63(10), 38 (2010).

[2] M. Fiebig, J. Phys. D: Appl. Phys. 38, R123 (2005).

[3] S. W. Cheong and M. Mostovoy, Nat. Mater. 6, 13 (2007).

[4] J. F. Scott, J. Mater. Chem. 22, 4567 (2012).

[5] D. Khomskii, Physics 2, 20 (2009).

[6] J. Wang, J. B. Neaton, H. Zheng, V. Nagarajan, S. B. Ogale, B. Liu, D. Viehland, V. Vaithyanathan, D. G. Schlom, U. V. Waghmare, N. A. Spaldin, K. M. Rabe, M. Wuttig, and R. Ramesh, Science 299, 1719 (2003).

[7] T. Kimura, Y. Sekio, H. Nakamura, T. Siegrist, and A. P. Ramirez, Nat. Mater. 7, 291 (2008).

[8] Z. Wang, N. Qureshi, S. Yasin, A. Mukhin, E. Ressouche, S. Zherlitsyn, Y. Skourski, J. Geshev, V. Ivanov, M. Gospodinov, and V. Skumryev, Nat. Commun. 7, 10295 (2016).

[9] Y. K. Jeong, J.-H. Lee, S.-J. Ahn, S.-W. Song, H. M. Jang, H. Choi, and J. F. Scott, J. Am. Chem. Soc. 134, 1450 (2012).

[10] S. Mukherjee, A. Roy, S. Auluck, R. Prasad, R. Gupta, and A. Garg, Phys. Rev. Lett. 111, 087601 (2013).

[11] Y. K. Jeong, J.-H. Lee, S.-J. Ahn, and H. M. Jang, Chem. Mater. 24, 2426 (2012).

[12] Z. Cheng, F. Hong, Y. Wang, K. Ozawa, H. Fujii, H. Kimura, Y. Du, X. Wang, and S. Dou, ACS Appl. Mater. Interfaces 6, 7356 (2014).

[13] J.-H. Lee, Y. K. Jeong, J. H. Park, M.-A. Oak, H. M. Jang, J. Y. Son, and J. F. Scott, Phys. Rev. Lett. 107, 117201 (2011).

[14] C. Y. Kuo, Y. Drees, M. T. Fernandez-Diaz, L. Zhao, L. Vasylechko, D. Sheptyakov, A. M. T. Bell, T. W. Pi, H. J. Lin, M. K. Wu, E. Pellegrin, S. M. Valvidares, Z. W. Li, P. Adler, A. Todorova, R. Kuchler, A. Steppke, L. H. Tjeng, Z. Hu, and A. C. Komarek, Phys. Rev. Lett. 113, 217203 (2014).

[15] C. Zhang, M. Shang, M. Liu, T. Zhang, L. Ge, H. Yuan, and S. Feng, J. Alloys Compd. 665, 152 (2016).

[16] Y. Yang, H. Xiang, H. Zhao, A. Stroppa, J. Zhang, S. Cao, J. Íniguez, L. Bellaiche, and W. Ren, Phys. Rev. B 96, 104431 (2017).

[17] Y. Tokunaga, N. Furukawa, H. Sakai, Y. Taguchi, T. Arima, and Y. Tokura, Nat. Mater. 8, 558 (2009).

[18] Z. Y. Zhao, X. M. Wang, C. Fan, W. Tao, X. G. Liu, W. P. Ke, F. B. Zhang, X. Zhao, and X. F. Sun, Phys. Rev. B 83, 014414 (2011).

[19] A. Stroppa, M. Marsman, G. Kresse, and S. Picozzi, New J. Phys. 12, 093026 (2010).

[20] Y. Tokunaga, S. Iguchi, T. Arima, and Y. Tokura, Phys. Rev. Lett. 101, 097205 (2008).
[21] W. C. Koehler, E. O. Wqllam, and M. K. Wilkinson, Phys. Rev. 118, 58 (1960).

[22] S. Bujko, D. Georgiev, K. Krezhov, V. Nietz, and G. Passage, J. Phys.: Condens. Matter. 7, 8099 (1995).

[23] I. Mareshal and J. Sivardicre, J. Phys 30, 967 (1969).

[24] T. Chatterji, M. Meven, and P. J. Brown, AIP Adv 7, 045106 (2017).

[25] T. Chatterji, A. Stunault, and P. J. Brown, J. Phys. Condens.: Mater 29, 385802 (2017).

[26] A. Bhattacharjee, K. Saito, and M. Sorai, J. Phys. Chem. Solids 63, 569 (2002).

[27] M. Shao, S. Cao, Y. Wang, S. Yuan, B. Kang, J. Zhang, A. Wu, and J. Xu, J. Cryst. Growth 318, 947 (2011).

[28] O. Nikolov, I. HaIl, and K. W. Godfrey, J. Phys.: Condens. Matter 7, 4949 (1995).

[29] J. Archer and M. S. Lehmann, J. Appl. Crystallogr. 19, 456 (1986).

[30] G. J. McIntyre and R. F. D. Stansfield, Acta Crystallogr. Sect. A 44, 257 (1988).

[31] J. C. Matthewman, P. Thompson, and P. J. Brown, J. Appl. Crystallogr. 15, 167 (1982).

[32] J. Strempfer, S. Francoual, D. Reuther, D. K. Shukla, A. Skaugen, H. Schulte-Schrepping, T. Kracht, and H. Franz, J. Synchrotron Radiat. 20, 541 (2013).

[33] A. Ghosh, K. Dey, M. Chakraborty, S. Majumdar, and S. Giri, Europhys. Lett. 107, 47012 (2014).

[34] A. Indra, K. Dey, S. Majumdar, I. Sarkar, S. Francoual, R. P. Giri, N. Khan, P. Mandal, and S. Giri, Phys. Rev. B 95, 094402 (2017).

[35] A. Indra, K. Dey, J. K. Dey, S. Majumdar, U. RÃ¹/4tt, O. Gutowski, M. v. Zimmermann, and S. Giri, Phys. Rev. B 98, 014408 (2018).

[36] See Supplemental Material at http://link.aps.org/supplemental/ 10.1103/PhysRevB.100.214432 for details of the pyroelectric measurements and neutron results.

[37] N. Terada, Y. S. Glazkova, and A. A. Belik, Phys. Rev. B 93, 155127 (2016).

[38] T. N. M. Ngo, U. Adem, and T. T. M. Palstra, Appl. Phys. Lett. 106, 152904 (2015).

[39] R. Chen and Y. Kirsh, Analysis of Thermally Stimulated Process (Pergamon, New York, 1981).

[40] W. Liu and C. A. Randall, J. Am. Ceram. Soc. 91, 3245 (2008).

[41] T. Kimura, T. Goto, H. Shintani, K. Ishizaka, T. Arima, and Y. Tokura, Nature (London) 426, 55 (2003).

[42] For example, A. Indra, S. Mukherjee, S. Majumdar, O. Gutowski, M. v. Zimmermann, and S. Giri, Phys. Rev. B 100, 014413 (2019). 
[43] K. Dey, A. Karmakar, S. Majumdar, and S. Giri, Phys. Rev. B 87, 094403 (2013).

[44] K. Dey, S. Majumdar, and S. Giri, Phys. Rev. B 90, 184424 (2014).

[45] K. Dey, A. Karmakar, A. Indra, S. Majumdar, U. RÃ̄1/4tt, O. Gutowski, M. v. Zimmermann, and S. Giri, Phys. Rev. B 92, 024401 (2015).

[46] S. Horiuchi, R. Kumai, Y. Okimoto, and Y. Tokura, Phys. Rev. Lett. 85, 5210 (2000).

[47] J. Koo, C. Song, S. Ji, J.-S. Lee, J. Park, T.-H. Jang, C.-H. Yang, J.-H. Park, Y. H. Jeong, K.-B. Lee, T. Y. Koo, Y. J. Park, J.-Y. Kim, D. Wermeille, A. I. Goldman, G. Srajer, S. Park, and S.-W. Cheong, Phys. Rev. Lett. 99, 197601 (2007).

[48] A. Indra, K. Dey, A. Midya, P. Mandal, O. Gutowski, U. Rütt, S. Majumdar, and S. Giri, J. Phys.: Condens. Matter 28, 166005 (2016).
[49] B. J. Campbell, H. T. Stokes, D. E. Tanner, and D. M. Hatch, J. Appl. Crystallogr. 39, 607 (2006).

[50] S. T. Bramwell, S. G. Carling, C. J. Harding, K. D. M. Harris, B. M. Kariuki, L Nixon, and I. P. Parkin, J. Phys.: Condens. Matter 8, L123 (1996).

[51] In $P b n 2_{1}$ space group three oxygens (O1, O2, and O3) exist.

[52] M. V. Berry, Proc. R. Soc. London Ser. A 392, 45 (1984).

[53] S. Mahana, B. Rakshit, R. Basu, S. Dhara, B. Joseph, U. Manju, S. D. Mahanti, and D. Topwal, Phys. Rev. B 96, 104106 (2017); 97, 224107 (2018).

[54] J. Sannigrahi, S. Bhowal, S. Giri, S. Majumdar, and I. Dasgupta, Phys. Rev. B 91, 220407(R) (2015).

[55] G. Yahia, F. Damay, S. Chattopadhyay, V. Baledent, W. Peng, S. W. Kim, M. Greenblatt, M. B. Lepetit, and P. FouryLeylekian, Phys. Rev. B 97, 085128 (2018). 